\title{
Carta
}

\section{MORTALIDAD MATERNA EN CHILE 1990-1999}

El interesante análisis de la mortalidad materna en Chile desarrollado por el autor Dr. Enrique Donoso $(1,2,3)$ concluye en una hipótesis demográfica para explicar el deterioro del indicador en 1999.

El factor demográfico, detectado en el estudio, es el aumento de nacimientos en mujeres de mayor edad (35-40 años), grupo etario de mayor riesgo obstétrico que debería ser considerado como tal en estrategias futuras orientadas a reducir la mortalidad materna.

Sin embargo, este planteamiento puede ser confundente si no considera que el cuidado médico es el intermediario entre un indicador pronóstico y la muerte materna. Además, la evidencia define con precisión la importancia del factor edad en relación con las causales de muerte certificadas.

La interpretación es que una mejoría continua del cuidado médico ofrecido a las pacientes estaría previniendo muertes maternas, explicando así la tendencia al descenso observada durante la década, pero limitaciones en este proceso estarían postergando mejores resultados.

Analizando a modo de ejemplo, en el grupo de muertes maternas por hemorragia (incluyendo embarazo ectópico) observamos 119 fallecimientos por esta causa en el período 90-99. Considerando que el $90 \%$ de estos casos son prevenibles (4), 107 decesos pudieron evitarse.

La distribución de estas muertes para dos quinquenios, 1990-1994 y 1995-1999 es similar (13,8 y $13,3 \%$ del total) y sugiere que las muertes por hemorragia durante la década no han cambiado en forma significativa.

Estudios recientes evalúan la calidad de atención de las madres con hemorragia obstétrica enfocándose en los procedimientos del cuidado médico y no en un indicador pronóstico (5).

En esa experiencia, en un país desarrollado con menor mortalidad materna y mejor calidad de atención, la aplicación de criterios definidos en la evaluación de la asistencia médica demostró que hasta un $38 \%$ de las pacientes con hemorragia obstétrica grave recibieron un cuidado médico insuficiente y por ende, susceptible de mejorar.

De los resultados de esa experiencia hacemos una doble interpretación:

1. Que en los países con mejor nivel de desarroIlo aún hay brechas superables en la prevención de muertes maternas por hemorragia, y

2. Que la promoción de estas estrategias en un medio con menor desarrollo tiene un gran potencial en la mejoría del indicador.

¿Cómo influye la edad materna en la incidencia de Desprendimiento Prematuro de Placenta (DP) y Placenta Previa (PP), dos causas importantes de hemorragia?

El concepto histórico es que mayor edad y mayor paridad se asocian con DP más frecuente (6), sin embargo en las series tradicionales no se consideró la influencia separada de ambas variables y tampoco se analizó los grupos extremos de edad y paridad. La evidencia actual en cambio demuestra que no hay aumento del DP con la mayor edad materna, no hay aumento con la mayor paridad sobre los 30 años, la gran multípara no tiene mayor riesgo de DP y que el riesgo es mayor cuando el tercer embarazo ocurre antes de los 20 años $(7,8)$.

Cinco de los 22 casos de muerte por hemorragia para 1998-1999 corresponden a DP (no hay certificación por esta causa en años anteriores). Sabemos que el DP es una patología compleja con un serio impacto perinatal y de morbilidad en la madre, pero la muerte materna es rara o infrecuente y la conclusión es que el cuidado médico en estos casos fue insuficiente, sea en el reconocimiento precoz o en el manejo de la forma más grave de esta patología.

En PP, a diferencia del DP, la edad materna mayor de 40 años constituye un factor de riesgo (OR 2,9) (7), sin embargo el incremento real de esta patología está en relación directa con la mayor 
frecuencia de operación cesárea (9) y la cesárea anterior es un factor de riesgo independiente para placenta acreta y cesárea-histerectomía y son estos casos los que concentran la mayor morbilidad y riesgo de muerte maternal $(10,11)$.

Un análisis similar puede hacerse para la hemorragia postparto, la hipertensión y otras causales certificadas.

Sin duda que hay complicaciones obstétricas asociadas a la mayor edad, pero la importancia del factor edad en su manejo ha sido confundente (12).

En resumen el planteamiento es que el objetivo de la vigilancia epidemiológica, más que una meta específica, es mantener un continuo en la tendencia observada del indicador durante la década pasada.

Con ese propósito sería beneficioso implementar un Comité de expertos que genere criterios específicos de calidad del cuidado médico de acuerdo a las posibilidades locales y que desarrolle el análisis permanente de los procedimientos de atención para las causales certificadas, optimizando además tanto la Auditoría como la Certificación.

\section{BIBLIOGRAFIA}

1. Donoso E, Poblete A, Villarroel L: Mortalidad Materna. Chile 1990-1996. Rev Chil Obstet Ginecol 2000; 63(4): 290-97.

2. Donoso E, Beker J, Villarroel L: Riesgo reproductivo en la quinta década de la vida. Mortalidad Materna. Chile 1999. XXIX Congreso Chileno de Obstetricia y Ginecología, Libro de Contribuciones, pág 155, C-85.

3. Donoso E: Mortalidad materna en Chile: tras el cumplimiento de una meta. Rev ChIL Obstet Ginecol 2002; 67(1): 44-6.
4. Bouvier-Colle MH, Varnoux N, Bréart G: The maternal deaths in France. Paris: Les Editions INSERM, 1994.

5. Bouvier-Colle MH, Dahada Ould El Joud, Varnoux N, Goffinet $\mathrm{F}$ et al: Evaluation of the quality of care for severe obstetrical haemorrhage in three french regions. Br J Obtest Ginaecol 2001; 108: 893-903.

6. Karegard M, Gennser G: Incidence and recurrence rate of abruptio placentae in Sweden. Obstet Gynecol 1986; 67: 523-8.

7. Ananth CV, Wilcox AJ, Savitz DA, Bowes W et al: Effect of maternal age and parity on the risk of uteroplacental bleeding disorders in pregnancy. Obstet Gynecol 1996; 88: 511-16.

8. Toohey JS, Keegan KA, Morgar MA et al: The dangerous multipara: Fact or fiction? Am J Obstet Gynecol 1995; 172: 683-6.

9. Singh PM, Rodríguez C, Gupta AN: Placenta previa and previous cesarean section. Acta Obstet Gynecol Scand 1981; 60: 367-8.

10. Clark SL, Yeh SY, Phelan JP, Bruce S, Paul RH: Emergency hysterectomy for obstetric hemorrhage. Obstet Gynecol 1984; 64: 376-80.

11. Marilyn C, Frederiksen, Glassenberg R, Stika C: Placenta previa: A 22-years analysis. Am J Obstet Gynecol 1999; 180: 1432-7.

12. Bell JS, Campbell DM, Graham WJ, Penney GC: Can obstetric complications explain the high levels of obstetric interventions and maternity services use among older women? A retrospective analysis of routinely collected data. Br J Obstet Gynaecol 2001; 108: $910-18$.

Dr. Jorge Hasbún H. Hospital J. J. Aguirre Universidad de Chile 